\title{
EL ENCUENTRO SOCIO-DEPORTIVO COMO EXPERIENCIA DE APRENDIZAJE-SERVICIO UNIVERSITARIO EN DOS CENTROS DE INTERNAMIENTO EDUCATIVO DE MENORES
}

\author{
Jorge Miguel Fernández Cabrera \\ Antonio Gómez Rijo \\ Francisco Jiménez Jiménez \\ Universidad de La Laguna \\ Merche Ríos Hernández \\ Universidad de Barcelona
}

\begin{abstract}
RESUMEN: En este estudio comparativo se presenta una misma experiencia de aprendizaje-servicio universitario llevada a cabo en el Centro de Internamiento Educativo de Menores Valle Tabares (CIEM VT) y en el Centre Educatiu de Justícia Can Llupià (CEJ CLL). Desde dos asignaturas de dos titulaciones distintas, una de la Universidad de La Laguna y otra de la Universitat de Barcelona, se organizó un encuentro sociodeportivo, que consistió en el diseño y práctica conjunta de retos cooperativos de carácter lúdico-motriz por parte de dos colectivos: alumnado universitario y personas privadas de libertad (PPL). Con el propósito de conocer la percepción de las personas participantes sobre la experiencia, se siguió un enfoque cualitativo mediante la aplicación de un cuestionario con preguntas abiertas y cerradas al alumnado universitario y una entrevista grupal a las PPL. Los resultados apuntan hacia una mejora del desarrollo de habilidades profesionales y la superación de estereotipos sociales por parte del alumnado universitario; y al empoderamiento de las PPL, al asumir responsabilidades personales y sociales (Deeley, 2016). Se constatan similitudes en la percepción del alumnado universitario con relación a las dimensiones 'vivencia' y 'aprendizaje', y en las respuestas de las entrevistas grupales realizadas a las PPL de ambos centros.
\end{abstract}

PALABRAS CLAVE: aprendizaje-servicio universitario, justicia juvenil, encuentro socio-deportivo, Educación Física. 


\title{
THE SOCIO-SPORTS MEETING AS AN EXPERIENCE OF LEARNING- UNIVERSITY SERVICE IN TWO CENTERS OF EDUCATIONAL INTERNATION FOR MINORS
}

\begin{abstract}
This comparative study presents the same university servicelearning experience carried out by the Valle Tabares Center for Educational Internment of Minors (CIEM VT) and the Center Educatiu de Justícia Can Llupià (CEJ CLL). From two subjects of two degrees, one from the University of La Laguna and the other from the University of Barcelona, a socio-sports meeting was organized, which consisted in the design and joint practice of cooperative challenges of a recreational-motor nature by two groups: university students and persons deprived of liberty (PPL). In order to know the perception of the participants about the experience, a qualitative approach was followed by applying a questionnaire with open and closed questions to university students and a group interview with PPL. The results point towards an improvement in the development of professional skills and the overcoming of social stereotypes by university students; and to the empowerment of PPL, by assuming personal and social responsibilities. There are similarities in the perception of university students in relation to the 'experience' and 'learning' dimensions, and in the responses of the group interviews carried out to the PPL of both centers.
\end{abstract}

KEYWORDS: University service-learning, juvenile justice, socio-sports meeting, Physical Education.

Recibido: $30 / 04 / 2020$

Aceptado: 03/07/2020

Correspondencia: Jorge Miguel Fernández Cabrera, Universidad de la Laguna, Facultad de Educación, Pabellón de Gobierno, C/ Molinos de Agua s/n, 38200 San Cristóbal de La Laguna, Santa Cruz de Tenerife. Email: mferca@ull.edu.es

\section{INTRODUCCIÓN}

Una de las funciones de la enseñanza universitaria es la de promover el compromiso social de su alumnado a través del desarrollo de iniciativas que combinen el aprendizaje académico con el servicio a la comunidad, orientado a la mejora de la calidad de vida y a la inclusión social. Para Martínez-Usarralde, Gil-Salom y Macías-Mendoza (2019), este proceso se puede organizar a través de un proyecto de aprendizaje-servicio (ApS), que se justifica por el significado social que se promueve en los aprendizajes académicos y la responsabilidad social que adquiere el estudiante universitario en su desarrollo.

El ApS como modelo pedagógico puede favorecer este compromiso, al proponer la aplicación de competencias curriculares en contextos y experiencias reales que permitan un beneficio social (Furco y Billig, 2002). Además, se trata de una metodología que mejora la motivación, el compromiso académico del alumnado univer- 
sitario (Billig, Root y Jesse, 2005), y el desarrollo de valores como la solidaridad, la cooperación y el trabajo en equipo (Chiva-Bartoll, Lidon y Salvador-García, 2020b; Ruiz-Montero, Chiva-Bartoll, Salvador-García y González-García, 2020); incrementándose, a la vez, la responsabilidad personal y social (Deeley, 2016; Tapia, 2008). El ApS se interesa, por tanto, en el desarrollo de competencias sociales que permitan atender conjuntamente a distintas problemáticas de la comunidad, para mejorarla y transformarla (Aubert, Bizkarra y Calvo, 2014; Gil-Gómez y Chiva-Bartoll, 2014; Santos-Rego, Sotelino y Lorenzo, 2015). También puede fomentar la superación de barreras que promuevan la inclusión social (Ríos, 2017), mediante la consideración del aprendizaje como un servicio a colectivos vulnerados. Sin olvidar que, también, estos colectivos aportan claves de aprendizaje relevante al alumnado universitario (Chiva-Bartoll, Capella-Peris y Salvador-García, 2020a; Ruiz-Montero et al., 2020); de tal forma que ambos, aprendizaje y servicio, se enriquezcan mutuamente (ChivaBartoll, Ruiz-Montero, Capella-Peris y Salvador-García, 2020c).

Tomando como referencia el ApS en la educación superior (ApSU) como perspectiva metodológica innovadora (Rodríguez-Gallego, 2013), en el ámbito de la Actividad Física y el Deporte, se han venido desarrollando diversas experiencias. Entre ellas, se encuentran los encuentros socio-deportivos (ESD), cuyo origen formal se remonta a 1987 (Ríos, 2010), con la puesta en marcha de proyectos de intervención en diversos ámbitos (centros penitenciarios de personas adultas, centros de internamiento educativo de menores, y centros de salud mental) (Lleixà y Ríos, 2015; Ríos 2004, 2010). Todas estas experiencias, en sus múltiples contextos, tienen en común su voluntariedad, el compromiso y la colaboración, desde una perspectiva transversal, que favorece en cada estudiante la capacidad crítica, reflexiva, activa, dialéctica y comunicativa (Deeley, 2016; Ríos, 2010).

Los ESD se organizan con la intención de promover la implicación simultánea y compartida del alumnado universitario y las PPL. A través de estos ESD, se pretende mejorar el conocimiento del alumnado universitario acerca de la realidad de estos centros, generar un marco de reflexión sobre el papel que puede desempeñar la actividad física en el desarrollo de habilidades personales y sociales de las PPL, y romper con la concepción estigmatizadora que suele acompañar a estos centros (Ríos, 2015). El ESD promueve en los estudiantes un aprendizaje mediante la participación activa en experiencias de servicio, la aplicación de sus conocimientos y habilidades en situaciones de la vida real, y un tiempo estructurado para la reflexión y discusión (Aramburuzabala, Santos-Pastor, Chiva-Bartoll y Ruiz-Montero, 2019). De este modo, se genera una práctica de acción y reflexión social y académica (Deeley, 2016; Eyler y Giles, 1999; Gil-Gómez, 2012; Tinning y Ovens, 2009). También ofrece a las PPL la posibilidad de empoderamiento y estimulación de su capacidad de resiliencia (Billig, 2000; Furco, 2003), al delegar en ellas responsabilidades en el proceso de preparación y desarrollo de las actividades que conforman estos ESD.

Desde la perspectiva de los objetivos de desarrollo sostenible (ODS), una enseñanza de calidad (ODS4) se relaciona con la aplicación del aprendizaje en un contexto social real y la reflexión sobre sus consecuencias, aspectos que constituyen rasgos estructurales del ApS. En el ESD como experiencia de ApS se genera un entorno de 
interacción inclusivo en el que colectivos diversos se reconocen y participan como iguales, aspectos que se relacionan con la reducción de desigualdades (ODS 10).

En el desarrollo del ESD como experiencia de ApSU, pretendemos ofrecer al alumnado universitario la oportunidad de conocer un contexto educativo específico en el que desarrollar competencias profesionales y recibir feedback acerca del grado de adecuación a las necesidades de las PPL; propiciando una oportunidad de relación social y de desarrollo de habilidades personales para la mejora del aprendizaje. Para optimizar este proyecto en el futuro, también necesitamos identificar y valorar la incidencia de la experiencia en la percepción y expectativas de los agentes implicados (alumnado universitario y PPL).

\section{Metodo}

Este estudio se enmarca en una investigación cualitativa que adopta una perspectiva paradigmática constructivista (Del Rincón, Arnal, Latorre y Sans, 1995) e interpretativa (Cohen y Manion, 1990). Para Sparkes (1992), estos paradigmas son especialmente útiles si lo que se pretende es desvelar el significado socialmente construido por los participantes (supuesto ontológico). Utiliza grupos pequeños bajo un enfoque comparativo, ideográfico, hermenéutico y dialéctico (supuesto metodológico) y tiene un interés basado en la comprensión e interpretación que se valida y acuerda socialmente en la acción (Flick, 2010). Específicamente, podemos ubicarla en el tipo fenomenológico (Merleau-Ponty, 1994), dado que se trata de identificar y analizar los significados compartidos por estudiantes universitarios, PPL y profesorado de los centros que han participado en la experiencia de ApSU.

El estudio analiza una misma experiencia de ESD desarrollada en el CIEM VT (Tenerife) y en el CEJ CLL (Barcelona), desde las asignaturas de Aprendizaje y Enseñanza de la Educación Física (AEEF) (Especialidad de Educación Física del Máster de Formación del Profesorado de Educación Secundaria y Bachillerato, de la Universidad de La Laguna), y Deporte y Juego (DJ) (Grado de Educación Social de la Facultad de Educación de la Universitat de Barcelona). Para esta iniciativa, se contó con la colaboración la Fundación Canaria de Juventud IDEO, y la Secretària de Mesures Penals, Reinserció i Atenció a la Víctima de la Generalitat de Catalunya. El ESD consistió en el diseño y práctica conjunta, por parte del alumnado universitario y las PPL, de tareas lúdico-deportivas de carácter cooperativo, y la elaboración de un logo y lema para la actividad. El servicio del ApSU se focalizó en ofrecer un tiempo de interacción entre iguales, a través de la práctica conjunta de las tareas diseñadas por los dos colectivos en el proceso de preparación del ESD, donde las PPL pudieron ejercitar sus habilidades sociales y asumir responsabilidades ante personas externas a estos centros educativos.

En cuanto al proceso seguido para la realización del ESD, se han tenido en cuenta los elementos que conforman la esencia del ApS (Chiva-Bartoll et al., 2019):

1. El vínculo curricular con las asignaturas implicadas se estableció tomando como referencia determinadas competencias presentes en las respectivas guías docentes de $A E E F$ (diseñar y desarrollar espacios de aprendizaje con especial atención a la equidad, y la educación emocional y en valores), y DJ (diseñar, desarrollar y re- 
gular procesos de relación educativa fundamentados, contextualizados y transformadores). En el colectivo de PPL, los referentes fueron algunos de los objetivos del ámbito personal, relacional y/o formativo de los proyectos educativos del CIEM $V T$ (dotar al menor de los instrumentos y recursos necesarios para alcanzar un nivel adecuado de competencia social, haciéndolo partícipe de su propio proceso educativo y potenciando la responsabilidad de sus acciones), y del CEJ CLL (promover una educación inclusiva de calidad en contextos vulnerados, y favorecer la igualdad de género y la no discriminación).

2. Respecto a la reciprocidad entre aprendizaje y servicio, las competencias a desarrollar por el alumnado universitario se relacionan directamente con la naturaleza del servicio a ofrecer. También, los objetivos formativos referidos a las PPL encuentran en el ESD un entorno significativo para su desarrollo. Entorno en el que el alumnado universitario aplica sus habilidades profesionales, y recibe feedback acerca del grado de adecuación de las actividades desarrolladas a las necesidades de las PPL, y estas disfrutan de un escenario de interacción en el que asumir protagonismo y desarrollar habilidades personales y sociales. De la experiencia de ESD emana un aprendizaje y enriquecimiento compartido por ambos colectivos.

3. La interiorización del aprendizaje se inicia en la parte final del encuentro, y se continúa mediante la reflexión que se genera en la cumplimentación del cuestionario por parte del alumnado universitario, y de la entrevista grupal para las PPL.

4. La toma de decisiones del alumnado universitario y las PPL está presente en las tres fases de la experiencia. En la fase de 'preparación', con la organización de comisiones para el diseño y aplicación de las tareas prácticas a desarrollar. En la fase de 'implementación', con la presentación alternativa y la puesta en práctica, de manera conjunta, de los retos motrices cooperativos; $y$, en la fase de 'valoración de la experiencia', con la expresión de sus percepciones.

5. La necesidad real a la que se atiende se concreta en la falta de interacción social de las personas internas con personas del exterior durante su periodo de privación de libertad, y la limitación que esto supone para el desarrollo de habilidades sociales.

Teniendo en cuenta las consideraciones anteriores, el desarrollo del ESD se estructuró en las siguientes fases:

1. Fase de preparación: De manera simultánea, se introdujo al alumnado del Máster (AM) y del Grado de Educación Social (AES) en la metodología ApS y se iniciaron las gestiones con los responsables institucionales del CIEM VT y CEJ CLL. Fruto de ambas iniciativas, en cada caso se realizó una visita de un educador a la Universidad para informar de las características del programa educativo de ambos centros y de los protocolos que se siguen en la interacción con las PPL. Por último, se realizó una visita del AM y el AES al CIEM VT y CEJ CLL, respectivamente, para conocer las características de sus instalaciones y equipamientos, además de tener, en el caso del CEJ CLL, el primer contacto con las PPL. Por último, se organizaron las comisiones (subgrupos) para el diseño y la práctica de los retos colaborativos por parte de ambos colectivos: alumnado universitario y PPL. 
2. Fase de implementación: En el ESD se pusieron en práctica de manera conjunta, durante una hora y media, los retos motrices cooperativos que habían sido diseñados por los distintos colectivos participantes. Al finalizar, se pasó a compartir un refrigerio o merienda, para concluir con una valoración conjunta de la experiencia.

3. Fase de valoración de la experiencia: Para valorar la percepción del alumnado universitario, se aplicó un cuestionario con preguntas abiertas y cerradas. En la valoración de la percepción de las PPL se empleó una entrevista grupal.

Por último, indicar que la experiencia desarrollada coincide con los rasgos de una buena práctica docente (innovadora, efectiva, sostenible y replicable) identificados por Corchuelo (2015) y Gradaille y Caballo (2016).

\subsection{Participantes}

Las personas participantes en esta experiencia, se recogen en la tabla 1.

Tabla 1. Participantes en la experiencia de ApSU

\begin{tabular}{|c|c|c|c|c|}
\hline Grupo & Asignatura & Centro & $\mathbf{N}^{\circ}$ alumnos & $\mathbf{N}^{\mathbf{0}}$ alumnas \\
\hline AM & $E A E F$ & ULL & 7 & 6 \\
\hline AES & $D J$ & UB & 8 & 10 \\
\hline \multicolumn{2}{|c|}{ PPL } & CIEM VT & 7 & 5 \\
\cline { 3 - 5 } & & CEJ CLL & 10 & 7 \\
\hline
\end{tabular}

Tanto el AM como el AES han sido seleccionadas de manera intencional (Flick, 2010, 2014), cumpliendo el requisito de estar matriculado en la asignatura de $A E E F$ y $D$ J, respectivamente.

\subsection{Recogida de datos}

Los métodos utilizados para la recogida de datos cualitativos (Flick, 2015) fueron: un cuestionario ad hoc con 6 preguntas abiertas y 5 preguntas cerradas con respuesta tipo Likert con 5 niveles (nada, poco, algo, bastante y mucho) para el AM, utilizadas como referencia y apoyo para el análisis cualitativo, y una entrevista grupal no estructurada para las PPL (tabla 2).

Para garantizar la calidad del análisis de los datos cualitativos, se han tenido en cuenta los criterios de Guba (1989): la credibilidad (abordando el fenómeno desde diversos enfoques metodológicos y diversas fuentes de información), transferencia (describiendo de manera exhaustiva el procedimiento y la obtención de datos), dependencia (describiendo con detalle el contexto de intervención) y neutralidad; así como el uso de la triangulación múltiple (Flick, 2010) en cuanto a datos (contrastando las diversas fuentes: cuestionario y entrevistas grupales), investigadores (procedentes de dos universidades diferentes), teorías (sustantivas de los investigadores y de los participantes contrastadas con las formales) y métodos (combinando perspectivas de 
análisis cuantitativas y cualitativas). La recolección de datos verbales a través de estas técnicas se ha visto como una de las herramientas más eficaces para desvelar los entresijos del análisis, la comprensión y la valoración de las experiencias, sus significados e interpretaciones por parte de los participantes en la investigación (Kvale, 2011).

Tabla 2. Instrumentos de recogida de datos utilizados en la experiencia de ApSU y las personas participantes

\begin{tabular}{|c|c|c|}
\hline Instrumento & Descripción & Participantes \\
\hline Cuestionario & $\begin{array}{l}\text { Cuestionario con preguntas abiertas (¿Qué con- } \\
\text { sideras que has aprendido con esta experiencia? } \\
\text { respecto 1: Experiencia docente personal; 2: } \\
\text { Vivencia personal; 3: Conocimientos del colec- } \\
\text { tivo; 4: Trabajo en equipo; 5: Educadores-as) y } \\
\text { cerradas (Valora de } 1 \text {-más negativo- a } 5 \text {-más } \\
\text { positivo- las aportaciones del ESD, según los si- } \\
\text { guientes aspectos: 1: Experiencia docente perso- } \\
\text { nal; 2: Vivencia personal; 3: Conocimiento del } \\
\text { colectivo; 4: Trabajo en equipo; Educadores-as). }\end{array}$ & $\begin{array}{l}\text { AM y AES } \\
\text { (cumplimentado al fina- } \\
\text { lizar la experiencia de } \\
\text { ApSU) }\end{array}$ \\
\hline $\begin{array}{l}\text { Entrevista } \\
\text { grupal }\end{array}$ & $\begin{array}{l}\text { Entrevista con preguntas abiertas: ¿qué valora- } \\
\text { ción hacen de la experiencia?; ¿Qué aspectos in- } \\
\text { cluirías o cambiarías en el ESD?; Si tuvieras que } \\
\text { verbalizar con una palabra el ESD, ¿Cuál sería? }\end{array}$ & $\begin{array}{l}\text { PPL } \\
\text { CIEM VT y CEJ CLL } \\
\text { (realizadas al finalizar la } \\
\text { experiencia de ApSU) }\end{array}$ \\
\hline
\end{tabular}

\subsection{Procedimiento de análisis}

Para el análisis de los datos cuantitativos de los 5 ítems cerrados se utilizó el programa estadístico SPSS 21, mientras que para el análisis de los datos cualitativos hemos seguido las orientaciones de Gibbs (2012), con ayuda del programa Nvivo 12. En cuanto al proceso, se realizó una primera fase exploratoria, basada en el análisis de contenido de corte cuantitativo, donde se obtuvieron frecuencias de tópicos que nos dieron un indicio de posibles temáticas interesantes a investigar. Esto nos llevó a una aproximación al sistema de categorías cualitativo, con una estructura jerárquica de clasificación de elementos macro (dimensiones) y micro (categorías) (tabla 3). Esta herramienta ad hoc se elaboró a partir de una reflexión sobre las respuestas a las preguntas abiertas del cuestionario que se le administró al AM y AES. Se proponen cuatro dimensiones, que abarcan diversos ámbitos de intervención vinculados al ESD: 'aprendizaje', relacionada con el desarrollo profesional; 'vivencia', relacionada con la experiencia personal; 'aportaciones percibidas', vinculadas a la percepción de lo aportado a las PPL y, por último, 'conocimiento del contexto' y de los colectivos que han participado en la experiencia de ApSU. Para la segmentación del texto se ha seguido un criterio temático. Como unidad de contexto se eligió el ESD para todos los documentos personales. La codificación se realizó de manera inductiva, a partir del sistema de categorías basado en las teorías sustantivas de los participantes y los investigadores. Una vez concluido este proceso, se ha podido contrastar y complementar con las teorías formales a partir de la consulta bibliográfica. 


\section{Resultados}

Se presentan los resultados cuantitativos sobre la percepción del alumnado universitario (AM y AES) y, a continuación, los resultados cualitativos de ambos colectivos (alumnado universitario y PPL).

\subsection{Resultados cuantitativos}

Los valores de las respuestas a la pregunta 'valora las aportaciones del ESD según los siguientes aspectos' muestran puntuaciones medias altas y una gran sintonía en ambos grupos de estudiantes (AM y AES) (figura 1), excepto en el 'conocimiento del colectivo', donde el AM señala como una limitación la falta de conocimiento previo de las PPL del CIEM VT con las que iba a interactuar en el ESD.

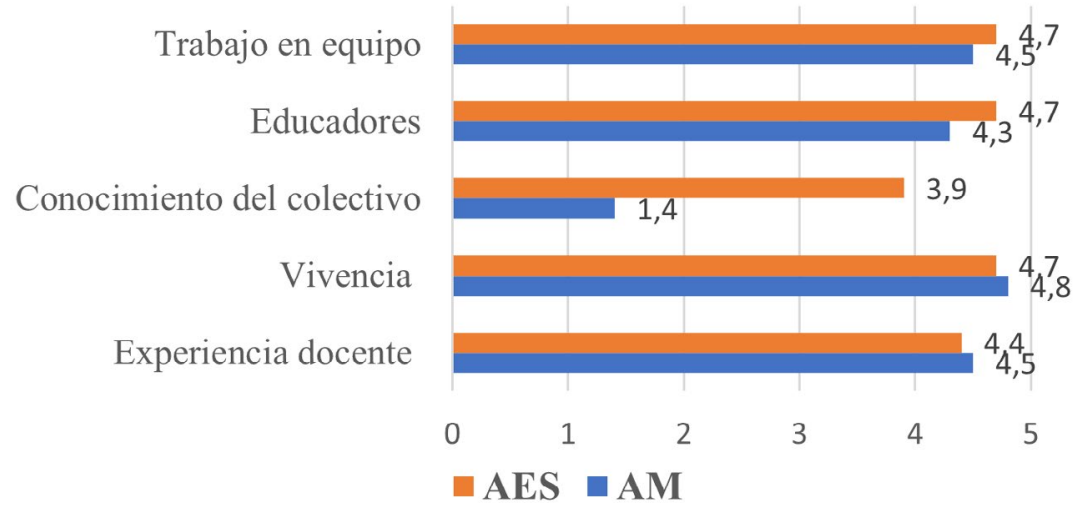

Figura 1. Resultados comparativos de los 5 ítems cerrados de cuestionario

\subsection{Resultados cualitativos}

Se presentan los resultados vinculados al análisis de los datos cualitativos relacionados con las preguntas abiertas del cuestionario que se le administró al AM y al AES y con las respuestas a la entrevista grupal realizada a las PPL del CIEM VT y CEJ CLL.

Percepción del alumnado universitario (AM y AES)

Para la valoración de las percepciones del AM y AES sobre los ítems de respuesta abierta del cuestionario, se presenta un perfil cuantitativo de las referencias por casos y los porcentajes de los datos globales extraídos de las dimensiones y categorías de la herramienta empleada en el análisis de contenido (tabla3). 
Tabla 3. Referencias y porcentajes por casos vinculados a cada dimensión y categoría

\begin{tabular}{|c|c|c|c|c|c|c|}
\hline \multirow{2}{*}{ Dimensiones } & \multirow{2}{*}{ Categorías } & \multirow{2}{*}{ Código } & \multicolumn{2}{|c|}{ AM (ULL) } & \multicolumn{2}{|c|}{ AES (UB) } \\
\hline & & & $\mathrm{N}=13$ & $\%$ & $\mathrm{~N}=18$ & $\%$ \\
\hline \multirow{7}{*}{ APRENDIZAJE } & Crear empatía con el alumnado & EMPA & 1 & 7,7 & 4 & 22,2 \\
\hline & Estrategias de motivación & MOTI & 3 & 23,1 & 2 & 11,1 \\
\hline & Trabajar de forma cooperativa & COOP & 8 & 61,5 & 10 & 55,6 \\
\hline & Estrategias de presentación & PRES & 2 & 15,4 & 3 & 16,7 \\
\hline & Trabajar de forma inclusiva & TINC & 3 & 23,1 & 3 & 16,7 \\
\hline & Potencial formativo actividad física & FOAF & 7 & 53,8 & 11 & 61,1 \\
\hline & $\begin{array}{l}\text { Diseño tareas para contexto espe- } \\
\text { cífico }\end{array}$ & COEX & 2 & 15,4 & 1 & 5,6 \\
\hline \multirow{5}{*}{ VIVENCIA } & $\begin{array}{l}\text { Experiencia gratificante y signifi- } \\
\text { cativa }\end{array}$ & GRAT & 11 & 84,6 & 14 & 77,7 \\
\hline & $\begin{array}{l}\text { Superar prejuicios y cambiar } \\
\text { mentalidad }\end{array}$ & PREJ & 10 & 76,9 & 12 & 66,7 \\
\hline & Deseo de repetir/más sesiones & REPE & 3 & 23,1 & 2 & 11,1 \\
\hline & Toma de contacto nuevo contexto & NUCO & 5 & 38,5 & 8 & 44,4 \\
\hline & Reto personal & RETO & 1 & 15,4 & 2 & 11,1 \\
\hline \multirow{3}{*}{$\begin{array}{l}\text { APORTACIONES } \\
\text { PERCIBIDAS }\end{array}$} & Buena convivencia y participación & COPA & 5 & 38,5 & 7 & 38,9 \\
\hline & Ayuda y mejora del grupo & AMEG & 3 & 23,1 & 2 & 11,1 \\
\hline & Compartir reflexiones finales & REFI & 1 & 7,7 & 4 & 22,2 \\
\hline \multirow[b]{2}{*}{$\begin{array}{l}\text { CONOCIMIENTO } \\
\text { CONTEXTO }\end{array}$} & Escaso conocimiento del contexto & $\mathrm{COCO}$ & 8 & 61,5 & 5 & 27.8 \\
\hline & $\begin{array}{l}\text { Buena implicación de los educa- } \\
\text { dores }\end{array}$ & IMED & 5 & 38,5 & 7 & 38,9 \\
\hline
\end{tabular}

Los resultados de porcentaje de ocurrencia en cada dimensión ponen de manifiesto que la experiencia de ApSU ha tenido un mayor impacto en las dimensiones 'vivencia' y 'aprendizaje', con porcentajes muy similares en ambos grupos de alumnado. A continuación, la dimensión 'conocimiento (previo) del contexto' y, con una presencia menor, la dimensión 'aportaciones percibidas' a las PPL. En cuanto a las categorías de las dimensiones, se observa que ambos grupos de alumnado universitario han coincidido en aquellas que han tenido un mayor porcentaje de ocurrencia en cada una de las dimensiones semánticas discriminadas, lo que viene a señalar que el ESD ha generado un efecto similar en los dos colectivos participantes. Como excepción, se constata que, en la categoría COCO, el grupo de AM reclama un mayor conocimiento previo del contexto antes del desarrollo del ESD, aspecto que ya fue señalado en las respuestas a los ítems cerrados del cuestionario. En cuanto a las divergencias en las categorías con menor porcentaje de ocurrencia, se observa que, en la dimensión 'aprendizaje', el AM ha planteado una mayor proporción de ocurrencias relacionados con las categorías MOTI, TINC y COEX, y el AES en la categoría EMPA y PRES; en la dimensión 'vivencia', el AM plantea una 
mayor proporción de ocurrencias en las categorías REPE y RETO; en la dimensión 'aportaciones percibidas', el AM plantea una mayor proporción de ocurrencias en la categoría AMEG, y el AES en REFI; y, por último, la divergencia ya comentada sobre la categoría COCO.

Desde una perspectiva más cualitativa, y por limitaciones de espacio, solo mostramos una selección de evidencias en cada dimensión que permitan ilustrar los resultados obtenidos en las categorías más relevantes (tablas 4, 5, 6, y 7), utilizando los correspondientes códigos para su identificación (tabla 3).

\section{Vivencia personal}

La participación en el ESD ha supuesto, para el alumnado de ambos grupos, una toma de contacto con un contexto social singular que no conocían y sobre el que tenían prejuicios. Su intervención en el mismo ha sido gratificante y ha tenido un gran impacto, que les ha demandado salir de su zona de confort, y les ha cambiado sus concepciones acerca de los centros de internamiento de menores y las personas que allí se encuentran (tabla 4).

Tabla 4. Evidencias de las categorías más relevantes de la dimensión 'Vivencia'

\begin{tabular}{|c|c|c|}
\hline Código & AM: $E A E F(\mathbf{N}=\mathbf{1 3})$ & AES: $D /(\mathbf{N}=18)$ \\
\hline $\begin{array}{l}\text { GRAT } \\
\text { (25 ocurrencias) }\end{array}$ & $\begin{array}{l}\text { - Ha sido una experiencia perso- } \\
\text { nal muy gratificante debido a que } \\
\text { nos ha sacado de nuestra "zona } \\
\text { de confort", ampliando nuestras } \\
\text { vivencias (C2) } \\
\text { (84,6\% de ocurrencias) }\end{array}$ & $\begin{array}{l}\text { - Ha sido una experiencia impactante } \\
\text { y gratificante (C1). } \\
\text {-Nunca había trabajado con este co- } \\
\text { lectivo y fue muy gratificante (C8) } \\
\text { ( } 77,7 \% \text { de ocurrencias) }\end{array}$ \\
\hline $\begin{array}{l}\text { PREJ } \\
\text { (22 ocurrencias) }\end{array}$ & $\begin{array}{l}\text {-He aprendido a quitarme pre- } \\
\text { juicios y miedos infundados (C3) } \\
\text {-He eliminado los prejuicios que } \\
\text { tenía anteriores a la experiencia } \\
\text { (C7) } \\
\text {-Mi pensamiento sobre estos } \\
\text { centros ha cambiado totalmente } \\
\text { después de esta experiencia (C8) } \\
\text { (76,9\% de ocurrencias) }\end{array}$ & $\begin{array}{l}\text {-El contacto con la realidad me ha } \\
\text { cambiado la manera de pensar sobre } \\
\text { estos centros (C6) } \\
\text {-Esta experiencia me ha servido para } \\
\text { eliminar estigmas sociales hacia este } \\
\text { colectivo (C8) } \\
\text {-Mi manera de ver estos centros ha } \\
\text { cambiado desde que he tenido la } \\
\text { oportunidad de tener una visión más } \\
\text { cercana (C13) } \\
(66,7 \% \text { de ocurrencias) }\end{array}$ \\
\hline $\begin{array}{l}\text { NUCO } \\
\text { (13 ocurrencias) }\end{array}$ & $\begin{array}{l}\text { - La novedad del contexto ha } \\
\text { contribuido a que la vivencia } \\
\text { haya tenido un mayor impacto } \\
\text { en el grupo (C4) } \\
(38,5 \% \text { de ocurrencias) }\end{array}$ & $\begin{array}{l}\text {-Este nuevo contexto inicial de falta } \\
\text { de libertad y estricta vigilancia me ha } \\
\text { causado un gran impacto, que se fue } \\
\text { superando con las experiencias de } \\
\text { relación en la práctica (C2) } \\
\text { (44,4\% de ocurrencias) }\end{array}$ \\
\hline
\end{tabular}




\section{Aprendizaje}

La participación en el diseño y desarrollo del ESD ha permitido al alumnado universitario de ambos grupos poner en valor y reforzar determinados aspectos de su formación profesional, como la toma de conciencia de la potencialidad de las dinámicas cooperativas para favorecer la interacción social, y la constatación de las posibilidades formativas de la actividad física en el desarrollo de valores y de responsabilidades personales y sociales (tabla 5).

Tabla 5. Evidencias de las categorías más relevantes de la dimensión 'Aprendizaje'

\begin{tabular}{|c|c|c|}
\hline Código & AM: $E A E F$ & AES: DJ \\
\hline $\begin{array}{l}\text { COOP } \\
\text { (18 ocurrencias) }\end{array}$ & $\begin{array}{l}\text { - La aplicación de retos coopera- } \\
\text { tivas favoreció la participación de } \\
\text { los internos (C11) } \\
(61,5 \% \text { de ocurrencias) }\end{array}$ & $\begin{array}{l}\text {-El trabajo en equipo favoreció el } \\
\text { acercamiento a los jóvenes con pri- } \\
\text { vación de libertad (C7) } \\
\text { (56,6\% de ocurrencias) }\end{array}$ \\
\hline $\begin{array}{l}\text { FOAF } \\
\text { (18 ocurrencias) }\end{array}$ & $\begin{array}{l}\text {-Por medio de la Educación Físi- } \\
\text { ca pudimos llegar al alumnado y } \\
\text { formarlo en valores (C9). } \\
\text { - La participación en actividades } \\
\text { físicas ha permitido conectar y } \\
\text { favorecer la relación con los in- } \\
\text { ternos (C12) } \\
(53,8 \% \text { de ocurrencias) }\end{array}$ & $\begin{array}{l}\text {-Se reforzó mi idea de que con el } \\
\text { deporte se pueden inducir valores } \\
\text { (C16) } \\
\text { - Las propuestas de actividad física } \\
\text { han mejorado el compañerismo y el } \\
\text { respeto (C18) } \\
\text {-Hemos comprobado la utilidad del } \\
\text { juego como herramienta educativa } \\
\text { (C15) } \\
(61,1 \% \text { de ocurrencias) }\end{array}$ \\
\hline
\end{tabular}

Aportaciones percibidas

En cuanto a la percepción de lo aportado a las PPL, se enfatizó el clima positivo generado durante los respectivos ESD (tabla 6).

Tabla 6. Evidencias de las categorías más relevantes de la dimensión 'Aportaciones percibidas'

\begin{tabular}{|c|c|c|}
\hline Código & AM: $E A E F$ & AES: DJ \\
\hline $\begin{array}{l}\text { COPA } \\
\text { (12 ocurrencias) }\end{array}$ & $\begin{array}{l}\text { - La motivación y al ánimo ayudó } \\
\text { a generar entusiasmo en el alum- } \\
\text { nado a la hora de participar en } \\
\text { los juegos (C10). } \\
\text { - Un día diferente, motivante. To- } \\
\text { dos salimos ganando (C2) } \\
(38,5 \% \text { de ocurrencias) }\end{array}$ & $\begin{array}{l}\text { - He estado muy a gusto, se puede } \\
\text { trabajar perfectamente con ellos y } \\
\text { tienen muchas ganas de jugar y apor- } \\
\text { tar ideas (C3) } \\
\text { - Gran acogida realizada por los jó- } \\
\text { venes del centro, mostrándose muy } \\
\text { cercanos y atentos durante toda la } \\
\text { sesión (C6) } \\
(38,9 \% \text { de ocurrencias) }\end{array}$ \\
\hline
\end{tabular}




\section{Conocimiento del contexto}

Por lo que se refiere al conocimiento del contexto de intervención, se señaló la buena implicación y disponibilidad de los educadores del CIEM VT y del CEJ CAN $L L$ y, como aspecto a mejorar, la información y conocimiento de las PPL antes de la celebración del ESD, más acentuado este aspecto en el caso del AM (tabla 7).

Tabla 7. Evidencias de las categorías más relevantes de la dimensión 'Conocimiento del contexto'

\begin{tabular}{|c|c|c|}
\hline Cód & AM: $E A E F$ & AES: DJ \\
\hline $\begin{array}{l}\text { COCO } \\
\text { (13 ocurrencias) }\end{array}$ & $\begin{array}{l}\text {-No hubo suficiente conoci- } \\
\text { miento del colectivo con el que } \\
\text { íbamos a trabajar. Solo tuvimos } \\
\text { conocimiento previo del grupo a } \\
\text { través de una charla (C11). } \\
\text {-El conocimiento del colectivo } \\
\text { ha sido limitado, ya que en la pri- } \\
\text { mera visita solo vimos las instala- } \\
\text { ciones (C9) } \\
\text {-Mi predisposición era dubitativa } \\
\text { ya que no sabíamos qué es lo que } \\
\text { nos íbamos a encontrar (C1) } \\
\text { (61,5\% de ocurrencias) }\end{array}$ & $\begin{array}{l}\text {-Aunque mi conocimiento sobre el } \\
\text { colectivo no es mucho, necesitan tra- } \\
\text { bajar como gestionar las emociones } \\
\text { y mejorar la autoestima (C8) } \\
\text {-Conocer al colectivo hace que tu } \\
\text { aportación sea más acertada y cer- } \\
\text { cana (C1) } \\
\text {-Pensaba que los centros cerrados } \\
\text { eran como cárceles. Con la experien- } \\
\text { cia nos dimos cuenta que no era así } \\
\text { (C9) } \\
\text {-Me ha dado la posibilidad de rom- } \\
\text { per esquemas, ya que no sabía que } \\
\text { me podía esperar en un centro de } \\
\text { estas características (C10) } \\
\text { (27,8\% de ocurrencias) }\end{array}$ \\
\hline $\begin{array}{l}\text { IMED } \\
\text { (12 ocurrencias) }\end{array}$ & $\begin{array}{l}\text { - Los educadores nos ayudaron y } \\
\text { nos facilitaron información para } \\
\text { la práctica (C4) } \\
\text {-Nos apoyaron en todo momen- } \\
\text { to y agradecieron nuestra ayuda } \\
\text { (C6) } \\
(38,5 \% \text { de ocurrencias) }\end{array}$ & $\begin{array}{l}\text { - He observado un trato muy cerca- } \\
\text { no por parte de los educadores (C1) } \\
\text { - La buena comunicación y coordina- } \\
\text { ción con los educadores ha favoreci- } \\
\text { do la marcha de la experiencia (C3) } \\
\text { (38,9\% de ocurrencias) }\end{array}$ \\
\hline
\end{tabular}

Percepción de las PPL (CIEM VT y CEJ CLL)

En los resultados de las entrevistas grupales con las PPL del CIEM VT y CEJ CLL (tabla 8), se constata un alto grado de satisfacción de la experiencia (GRAT), ya que ha sido muy positivo el conocer a gente nueva, que se fomente el compañerismo, la diversión y el trabajo en equipo. En este aspecto, coinciden con la opinión del AM y AES en las respuestas abiertas del cuestionario, que han valorado la experiencia como gratificante y significativa. También destaca, sobre todo en las PPL del CEJ CLL la superación de prejuicios (PREJ), que les ha generado esta experiencia, que coincide con la opinión del alumnado universitario en general, así como el efecto que ha tenido esta actividad física compartida en la alteración de su percepción 
temporal. Ambos colectivos de PPL manifiestan también el deseo de repetición (REPE) de la experiencia, cuestión que coincide con la opinión de parte del AM y AES. Asimismo, existe coincidencia en la mayoría de las palabras (PALA) mencionadas por las PPL del CIEM VT y CEJ CLL, valorando positivamente el encuentro. Por último, se realizan propuestas de mejora (MEJO), especialmente en el CIEM VT, solicitando un contacto con el AM antes de realizar el ESD y el aumento del tiempo de reflexión tras su finalización, para poder expresar mejor las vivencias personales relacionadas con el ESD.

Tabla 8. Evidencias de las categorías más relevantes en las entrevistas grupales a las PPL del CIEM VT y CEJ CLL

\begin{tabular}{|c|c|c|}
\hline Códigos & Personas internas CIEM VT & Personas internas $C E J C L L$ \\
\hline GRAT & $\begin{array}{l}\text {-Ha sido positivo conocer a nue- } \\
\text { va gente } \\
\text {-Nos hemos divertido mucho } \\
\text { con las actividades realizadas en } \\
\text { el encuentro } \\
\text {-Nos ha gustado mucho el tiem- } \\
\text { po de la merienda, después de } \\
\text { las actividades } \\
\text {-Me ha gustado trabajar en } \\
\text { equipo }\end{array}$ & $\begin{array}{l}\text {-Me ha encantado que viniesen los } \\
\text { estudiantes y que se fomente el com- } \\
\text { pañerismo y el deporte. Nos transmi- } \\
\text { ten cosas positivas } \\
\text {-Me han gustado los juegos, el buen } \\
\text { rollo, las personas que han venido. } \\
\text { Olvido que estoy aquí } \\
\text {-Ha habido confianza, como si los } \\
\text { conociera de siempre. Gracias por } \\
\text { venir }\end{array}$ \\
\hline PREJ & & $\begin{array}{l}\text {-Los estudiantes nos miran bien y } \\
\text { nos valoran } \\
\text {-Los estudiantes vienen, no nos } \\
\text { conocen, juegan con nosotros y } \\
\text { no nos juzgan. Saben que estamos } \\
\text { aquí por algo nada bueno; y, aun } \\
\text { así, vienen }\end{array}$ \\
\hline REPE & $\begin{array}{l}\text {-Nos gustaría realizar la activi- } \\
\text { dad periódicamente }\end{array}$ & $\begin{array}{l}\text { - Molaría que vinierais una vez por } \\
\text { semana, así pasaría más rápido el } \\
\text { tiempo }\end{array}$ \\
\hline PALA & $\begin{array}{l}\text {-Felicidad, repetir, amistad, liber- } \\
\text { tad, emoción, orgullo, compañe- } \\
\text { rismo diversión y alegría }\end{array}$ & $\begin{array}{l}\text {-Guapo, fantástico, diversión, ale- } \\
\text { gría, ilusión, emoción, tranquilidad, } \\
\text { igualdad, compañerismo, respeto, } \\
\text { vivir, buen rollo, vitalidad }\end{array}$ \\
\hline MEJO & $\begin{array}{l}\text {-Nos gustaría conocer a los estu- } \\
\text { diantes antes de iniciar la activi- } \\
\text { dad, para generar más confianza } \\
\text {-Proponemos aumentar el tiem- } \\
\text { po de reflexión posterior a la ac- } \\
\text { tividad. } \\
\text {-Proponemos repetir la actividad } \\
\end{array}$ & \\
\hline
\end{tabular}




\section{Discusión}

El análisis comparativo de una misma experiencia de ApSU en dos centros diferentes, ha evidenciado que el ESD puede ser considerado como una buena práctica de innovación educativa al haberse desarrollado una alternativa novedosa (innovadora), al demostrarse que ha tenido un impacto positivo (efectiva), al mantenerse en el tiempo y producir efectos duraderos (sostenible), y al poder aplicarse en otros contextos similares (replicable) (Corchuelo, 2015; Gradaille y Caballo, 2016; Ríos, 2010).

La similitud en los resultados de las preguntas cerradas del cuestionario y de las categorías que han acaparado una mayor frecuencia de ocurrencia en cada dimensión semántica del análisis de contenido de las preguntas abiertas, evidencian que el ApSU ha ofrecido al alumnado universitario un entorno real de aprendizaje privilegiado donde concienciarse, ser solidarios (Chiva-Bartoll et al., 2020b) y poder dar respuesta a una necesidad social (Furco y Billig, 2002). La aplicación del aprendizaje en un contexto social real y la reflexión sobre sus consecuencias sitúan el ApSU desarrollado en el contexto de una enseñanza de calidad (ODS4). El ESD también les ha permitido poner a prueba algunas competencias profesionales como la cooperación y el trabajo en equipo (Chiva-Bartoll et al., 2020b), aplicando y comprobando el alcance formativo de determinadas opciones de intervención docente (Billig et al., 2005; Chiva-Bartoll et al., 2020c; Chiva-Bartoll et al., 2020a). Asimismo, el disponer de un tiempo estructurado para la reflexión y discusión de la experiencia de servicio, ha posibilitado al alumnado universitario valorar su intervención (Aramburuzabala et al., 2019), y tomar conciencia de la necesidad de trascender sus prejuicios sobre el colectivo vulnerado con el que han compartido la experiencia. Así, el ApSU desarroIlado se ha configurado como una práctica de acción y reflexión (Deeley, 2016; Eyler y Giles, 1999; Gil-Gómez, 2012; Tinning y Ovens, 2009). Fruto de esta reflexión, el alumnado ha destacado que la estructura cooperativa de los retos lúdicos planteados ha favorecido la empatía entre los diversos colectivos participantes, y que este tipo de actividad física diseñada y desarrollada con un enfoque inclusivo de reconocimiento mutuo se muestra efectiva como herramienta educativa (Ríos 2017), induciendo el desarrollo de valores (Ruiz et al., 2020). El compañerismo y el trabajo en equipo también ha sido reconocido por las $\mathrm{PPL}$, aspecto que podríamos considerar como feedback positivo de lo realizado para el alumnado universitario. Este último colectivo también destaca el importante papel de las estrategias de presentación de las tareas, caracterizadas por el entusiasmo en la dinamización de la actividad, así como la incidencia de la actitud positiva, las orientaciones y los apoyos aportados por los educadores de los centros en el buen desarrollo del ESD.

Pero si el ESD ha tenido una incidencia formativa en el aprendizaje profesional, su efecto ha sido aún mayor en el plano personal del alumnado universitario y las PPL. El alumnado universitario ha tomado conciencia de la existencia de un colectivo social vulnerado, habitualmente estigmatizado, y sobre el que tenían prejuicios y miedos. Su contacto con él a través de esta experiencia de ApSU, les ha sacado de su zona de confort, les ha permitido conocerlos, reconocerlos y cambiar su percepción sobre él, descubriendo que interactuar con PPL a través de la actividad física les ha resultado una experiencia gratificante y con un gran potencial formativo (Ruiz et al., 
2020). Es evidente que la experiencia le ha exigido al alumnado hacer frente a sus nociones iniciales de alteridad, y les ha permitido transformarlas y construir nociones más reales de diversidad cultural, lo que sin duda refuerza su capacidad para trabajar en equipo y alcanzar objetivos comunes (Aramburuzabala et al., 2019), y los compromete con la reducción de desigualdades (ODS 10).

El carácter gratificante de la experiencia también ha sido manifestado por las PPL, matizando cuestiones como el trabajo en equipo, conocer a nuevas personas que les transmiten cosas positivas, sentirse valorados por el alumnado universitario sin ser juzgados, confirmando esa satisfacción mediante las palabras emitidas al final del encuentro. Estas mejoras en aspectos sociales y emocionales coinciden con las observadas en el estudio de Lleixà y Ríos (2015).

EI ESD ha generado expectativas de repetir la experiencia en ambos colectivos, por parte de las PPL del CEJ CLL incluso una vez por semana, reconociendo que su participación en ESD les ha alterado su percepción del tiempo ("olvido que estoy aquí), provocando una inconsistencia y ruptura temporal de la reclusión. Este aspecto ya ha sido señalado por estudios de otros autores en el ámbito de las prisiones (Fornons, 2008, Ríos 2004), que también ven que el ejercicio físico y el deporte pueden tener un papel de "evasión" simbólica de la experiencia cotidiana de las PPL mientras dura la condena (Martos-García, Devís-Devís y Sparkes, 2009).

Como aspectos a mejorar, señalados por el AM y PPL del CIEM VT, está la importancia de conocerse antes de llevar a cabo el encuentro.

Respecto a las limitaciones del estudio y aspectos a tener en cuenta para futuras investigaciones, se puede señalar el carácter puntual de la intervención. Aunque el proceso de preparación en el que ambos colectivos están implicados tiene una extensión mayor, sería interesante valorar la opción de realizar más de un ESD durante el desarrollo de las asignaturas y, si fuera posible, que alguno de ellos fuera desarrollado en las instalaciones universitarias. Asimismo, la estrategia de valoración de la experiencia debería considerar herramientas de recogida de datos de carácter más procesual (diario de campo) para una mejor comprensión de la experiencia y el ajuste de las dificultades que puedan surgir.

\section{Conclusiones}

EI ESD como experiencia de innovación que ha considerado los elementos que conforman la esencia del ApSU, supone una buena práctica educativa con potencial para ser transferida a otros contextos similares, como ha quedado acreditado en los resultados del estudio comparativo.

EI ESD ha ofrecido a las PPL una oportunidad de sentirse competentes en la asunción y desarrollo de responsabilidades personales y sociales ante otro grupo de personas (alumnado), compartiendo de manera inclusiva una actividad gratificante que les ha permitido romper la línea temporal de su internamiento y sentirse reconocidas.

El alumnado universitario ha dispuesto de un entorno de aprendizaje que le ha puesto en contacto con una realidad social desconocida, que les ha demandado salir 
de su zona de confort y superar estereotipos acerca de las personas con medidas judiciales, y con la labor que se realiza en los centros donde están internadas. Además, han tomado conciencia de las posibilidades formativas de la actividad física, de un nuevo ámbito de ejercicio profesional, y han aprendido a adaptar sus habilidades profesionales, desde un enfoque inclusivo, a un contexto singular.

\section{REFERENCIAS BIBLIOGRÁFICAS}

Aramburuzabala, P., Santos-Pastor, M. L., Chiva-Bartoll, O. y Ruiz-Montero, P. J. (2019). Perspectivas y retos de la intervención e investigación en aprendizajeservicio universitario en actividades físico-deportivas para la inclusión social. Publicaciones, 49(4), 19-27. http://doi.org/10.30827/publicaciones.v49i4.11726

Aubert, A., Biskarra, M. y Calvo, J. (2014). Actuaciones educativas de éxito desde la Educación Física. Retos. Nuevas Tendencias en Educación Física, Deporte y recreación, 25, 144-148.

Billig, S. H. (2000). Research on K-12 school-based service-learning: the evidence builds. Phi Delta Kappan, 81, 658-664.

Billig, S. H., Root, S. y Jesse, D. (2005). The impact of participation in service-learning on high school students' civic engagement. Circle Working Paper 33. College Park, MD: Center for Information and Research on Civic Engagement and Learning.

Corchuelo, C. (2015). Buenas prácticas docentes en contextos de exclusión social. Cuestiones pedagógicas, 24, 25-41.

Chiva-Bartoll, O., Capella-Peris, C. y Salvador-García, C. (2020a). Service-learning in physical education teacher education: towards a critical and inclusive perspective. Journal of Education for Teaching. http://doi.org/10.1080/02607476.2020.17 33400

Chiva-Bartoll, O., Lidon, M. y Salvador-García, C. (2020b). Can service-learning promote social well-being in primary education students? A mixed method approach. Children and Youth Services Review, 111, 1-8. http://doi. org/10.1016/j.childyouth.2020.10484

Chiva-Bartoll, O., Ruiz-Montero, P. J., Capella-Peris, C. y Salvador-García, C. (2020c). Effects of Service Learning on Physical Education Teacher Education Students' Subjective Happiness, Prosocial Behavior, and Professional Learning. Frontiers in Psychology, 11, 1-9. http://doi.org/10.3389/fpsyg.2020.00331

Chiva-Bartoll, O., Ruiz-Montero, P. J., Martín-Moya, R., Pérez-López, I., Giles-Girela, J., García-Suárez, J. y Rivera-García, E. (2019). University Service-Learning in Physical Education and Sport Sciences: A systematic review. Revista Complutense de Educación, 30(4), 1147-1164.

Cohen, L. y Manion, L. (1990). Métodos de investigación educativa. Madrid: La Muralla.

Deeley, S. (2016). El Aprendizaje-Servicio en educación superior. Teoría, práctica y perspectiva crítica. Madrid: Narcea. 
Del Rincón, D., Arnal, J., Latorre, A. y Sans, A. (1995). Técnicas de investigación en Ciencias Sociales. Madrid: Dykinson.

Eyler, J. y Giles, D. E. (1999). Where's the learning in service-learning? San Francisco: Jossey-Bass.

Flick, U. (2010). Introducción a la Investigación Cualitativa. Madrid: Morata.

Flick, U. (2014). La gestión de la calidad en Investigación Cualitativa. Madrid: Morata.

Flick, U. (2015). El diseño de Investigación Cualitativa. Madrid: Morata.

Fornons, D. (2008). La práctica Deportiva en la prisión: rehabilitación o evasión. En L. Cantarero, F. X. Medina y R. Sánchez (Eds.). Actualidad en el deporte: investigación y aplicación (pp. 215-227). Donosti: Ankulegi Antropologia Elkartea.

Furco, A. y Billig, S. H. (2002). Service Learning. The essence of the Pedagogy. Greenwich.

Furco, A. (2003). Issues of definition and programme diversity in the study of servicelearning. En Billig, S. H. y Waterman, A. S., Studying service-learning: Innovations in educational research methodology (pp. 13-34). Lawrence Erlbaum Associates.

Gibbs, G. (2012). El análisis de datos cualitativos en Investigación Cualitativa. Madrid: Morata.

Gil-Gómez, J. (2012). El aprendizaje-servicio en la enseñanza superior: una aplicación en el ámbito de la educación física. Tesis inédita de doctorado. Universitat Jaume I de Castellón.

Gil-Gómez, J. y Chiva-Bartoll, O. (2014). Una experiencia de aprendizaje-servicio en la asignatura 'Bases Biológicas y Fisiológicas del Movimiento' del Área de Didáctica de la Expresión Corporal. Retos. Nuevas Tendencias en Educación Física, Deporte y Recreación, 26, 123-128.

Gradaille, R. y Caballo, M. (2016). Las buenas prácticas como recurso para la acción comunitaria: criterios de identificación y búsqueda. Contextos educativos, 19, 7588. http://doi.org/10.18172/con.2773

Guba, E. G. (1989). Criterios de credibilidad en la investigación naturalista. En J. Gimeno y A. Pérez (Eds.), La enseñanza: su teoría y su práctica (pp. 148-165). Madrid: Akal.

Kvale, S. (2011). Las entrevistas en Investigación Cualitativa. Madrid: Morata.

Lleixà, T. y Ríos, M. (2015). Service-Learning in Physical Education Teacher Training. Physical Education in the Modelo Prison, Barcelona. Qualitative Research in Education, 4(2), 106-133. http://doi.org/10.17583/qre.2015.1138

Martínez-Usurralde, M. J., Gil-Salom, D. y Macías-Mendoza, D. (2019). Revisión sistemática de responsabilidad social universitaria y aprendizaje-servicio. Revista Mexicana de Investigación Educativa, 24(80), 149-172. 
Martos-García, D., Devís-Devís, J. y Sparkes, A. C. (2009b). Deporte entre rejas ¿Algo más que control social? Revista internacional de sociología, 67(2), 391-412. http:// doi.org/10.3989/RIS.2007.07.26

Merleau-Ponty, M. (1994). Fenomenología de la percepción. Barcelona: Península.

Ríos, M. (2004). La educación física en los establecimientos penitenciarios de Catalunya. Tàndem. Didáctica de la Educación Física, 15, 69-82.

Ríos, M (2010). La Educación Física en la Educación Social: una experiencia de aprendizaje y servicio. En M. Martínez (Ed.), Aprendizaje servicio y responsabilidad social de las universidades (pp. 113-128). Barcelona: Octaedro.

Ríos, M. (2015). La Educación Física como recurso de intervención socioeducativa. Los créditos ApS en centros penitenciarios y de salud mental. IPSE-ds, 8, 35-42.

Ríos, M. (2017). El deporte como recurso educativo en la inclusión social. Educació Social. Revista d'Intervenció Socioeducativa, 65, 44-56.

Rodríguez-Gallego, M. R. (2013). El Aprendizaje-Servicio como estrategia metodológica en la Universidad. Revista Complutense de Educación, 25, 95-113. http://doi. org/10.5209/rev_RCED.2014.v25.n1.41157

Ruiz-Montero, P., Chiva-Bartoll, O., Salvador-García, C. y González-García C. (2020). Learning with Older Adults through Intergenerational Service Learning in Physical Education Teacher Education. Sustainability, 12, 1-14. http://doi.org/10.3390/ su12031127

Santos-Rego, M. A., Sotelino, A. y Lorenzo, M. (2015). Aprendizaje-servicio y misión cívica de la universidad. Barcelona: Octaedro.

Sparkes, A. (1992). Breve introducción a los paradigmas de investigación alternativos en Educación Física. Perspectivas de la Actividad Física y el Deporte, 11, 29-33.

Tapia, M. N. (2008). La solidaridad como pedagogía. Buenos Aires: Criterio.

Tinning, R. y Ovens, A. (2009). Aprender a convertirse en un docente (en formación) reflexivo. En L. Martínez y R. Gómez (Coords.), La Educación Física y el deporte en la edad escolar. El giro reflexivo en la enseñanza (pp. 315-352). Buenos Aires: Miño Dávila. 